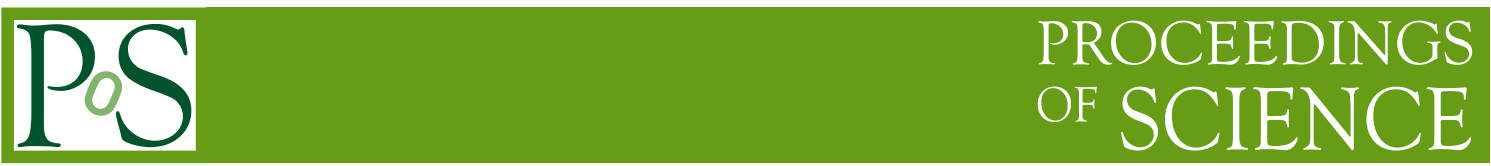

\title{
Theory Vision at LHCP 2019
}

\author{
Nathaniel Craig ${ }^{* \dagger}$ \\ University of California, Santa Barbara \\ E-mail: ncraig@physics.ucsb.edu
}

\begin{abstract}
Drawing upon the theory vision articulated at the LHC's inception, I survey the progress of the last 35 years and summarize the questions confronting particle physics in the present day. The discovery of the Higgs boson at the LHC illuminates the origin of mass but poses a host of new questions: Is the Higgs elementary, or composite? Does it interact with itself? Does it mediate a Yukawa force between fundamental particles? These are the questions of our era, and I argue that they define compelling experimental targets within reach of the HL-LHC and future Higgs factories, with answers that are profoundly interesting whether or not they agree with Standard Model predictions. Equally important is the LHC's role in exploring more speculative possibilities for physics beyond the Standard Model, from the nature of dark matter to the origin of flavor to the electroweak hierarchy problem. I emphasize the value of the hierarchy problem as an empiricallymotivated strategy for looking for new physics, highlighting novel possibilities motivated by LHC null results in conventional searches. Complementing specific theoretical motivations, a host of new search strategies, signatures, hardware, and detectors will maximize the LHC's sensitivity to unforeseen new physics in the coming years. I conclude by discussing the powerful role that proposed Higgs factories can play in answering the key questions of our era, emphasizing the value of exploration in making the physics case for current and future colliders.
\end{abstract}

7th Annual Conference on Large Hadron Collider Physics - LHCP2019

20-25 May, 2019

Puebla, Mexico

\footnotetext{
* Speaker.

${ }^{\dagger}$ I would like to thank the organizers of LHCP 2019 for an outstanding conference and the opportunity to articulate the ideas summarized here, as well as Indara Suarez, Frank Golf, and Francesco Pandolfi for enlightening follow-up.
} 


\section{Theory visions}

In contemplating a theory vision for the LHC circa 2019, I found it helpful to first revisit the theory vision for the LHC as it was articulated at the collider's inception. An exceptionally clear version of this vision is laid down in the Proceedings of the ECFA-CERN Workshop held in Lausanne and Geneva in March 1984 [1]. In the summary report, the organizers turn from the successes of particle physics in the 70's and early 80's to the new questions these successes posed:

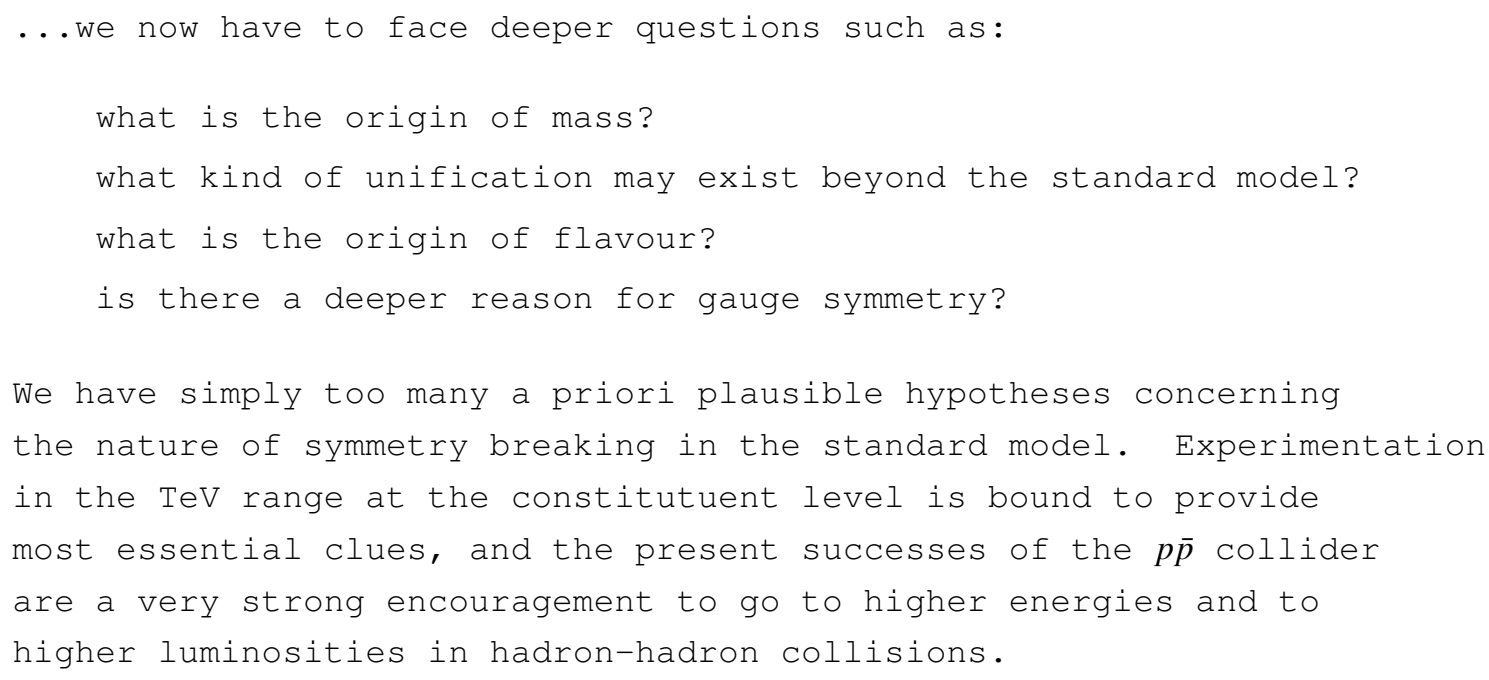

These questions formed the heart of the physics case for the LHC, and have driven considerable experimental and theoretical activity to this day. So where are we today? Of the original questions posed in 1984, we have answered one: the origin of mass appears to be (at least in part) the Higgs mechanism as manifest by the discovery of the Higgs boson. The other questions remain open, although abundant data has sharpened them considerably. Indeed, data from the LHC and a host of other experiments performed over the past 35 years have told us a great deal about what mechanisms don't underlie, say, the unification of forces beyond the Standard Model or the observed flavor structure. These experiments have also added or sharpened new questions. To the questions of 1984, I would also add two more that have come into greater relief in the ensuing years: the nature of dark matter and the apparent conservation of $\mathrm{CP}$ in the strong interactions. ${ }^{1}$ Although it is not obvious at first glance that the LHC is an appropriate machine to test these questions, as we will see, it has much to say. This leaves us with the following set of deep questions in 2019:

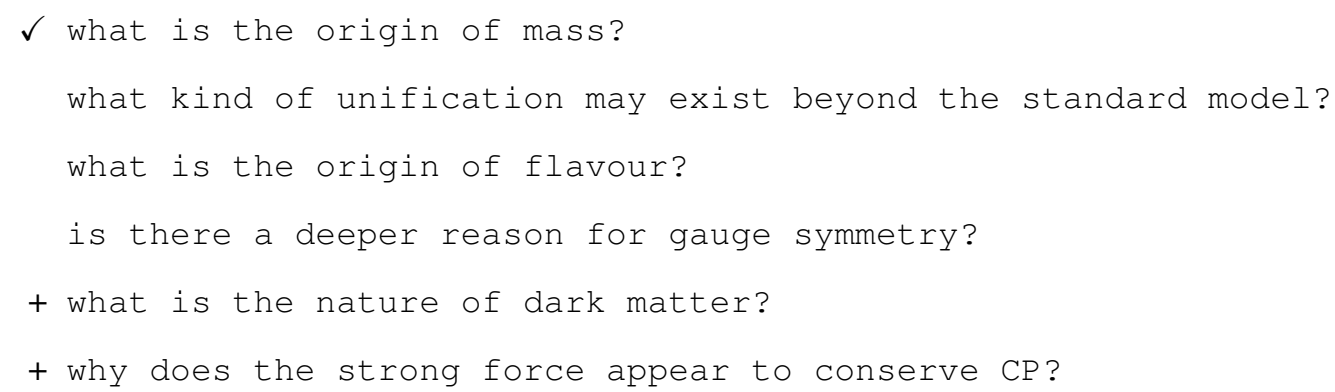

${ }^{1}$ Of course, one could imagine adding quite a few more, particularly involving the nature of gravity; I omit them here only for the sake of brevity. 
But much as the triumphs of the 70's and early 80's laid the groundwork for a whole new host of questions, the light cast on the origin of mass by the discovery of the Higgs boson in 2012 raises far more questions than it answers. Is the Higgs we've observed the only one, or simply one member of a larger Higgs sector responsible for electroweak symmetry breaking? Is it the Standard Model Higgs, or do its properties deviate from the Standard Model? Why is there electroweak symmetry breaking in the first place, i.e., why is $m_{H}^{2}$ negative? Given electroweak symmetry breaking, what sets the scale? These questions must now take their place alongside their long-standing predecessors, and play an influential role in both the future LHC and whatever comes thereafter.

\section{Building on discovery}

The discovery of the Higgs boson is one of the greatest scientific triumphs of the 21st century. But for all the excitement of its discovery, we still know very little about the Higgs. Broadly speaking, the question now is not whether the new state at $125 \mathrm{GeV}$ is $a$ Higgs, but whether it is the Higgs - the Higgs of the Standard Model. As is well known by now, the properties of the Standard Model Higgs are uniquely predicted given its mass, so that any deviations from Standard Model predictions are smoking-gun signatures of new physics. But, practically speaking, what does it mean to test whether the Higgs is the Standard Model Higgs? The best we can ever hope to do is to measure its properties in all available channels, and (in the absence of significant disagreement) verify consistency with Standard Model predictions to increasing precision. But but how precise is precise enough? What have we learned qualitatively by verifying agreement with the Standard Model at the ten percent level, the percent level, or the permille level?

I find the onward march of precision measurement itself to be somewhat unhelpful - it does not provide any qualitative benchmarks for achievement, and it places perhaps too great a value on the observation of deviations, rather than the confirmation of a beautiful paradigm. Thus, rather than focusing on the ever-increasing march of precision, it is perhaps more useful to reframe our study of the Higgs in terms of a few major conceptual questions. Each of these questions provides a motivated target for experiment, and an answer to any of these questions is of tremendous value regardless of the form it takes. These include:

- Is the Higgs elementary, or composite?

- Does the Higgs interact with itself?

- Does the Higgs mediate a Yukawa force?

To me, at least, these are the central questions of the post-discovery era. They provide sharp benchmarks for precision measurements of the Higgs. They are questions of fundamental significance about which the LHC can say a great deal. And perhaps most crucially, the answers to these questions are all profoundly interesting, whether or not they are in agreement with Standard Model predictions. 


\subsection{An elementary scalar?}

That the recently-discovered Higgs is a scalar is not remarkable in and of itself. We have seen scalars in nature already: the strong interaction provides them in abundance in the rich spectrum of scalar and pseudoscalar mesons. However, these scalars are all composite degrees of freedom, in the sense that they are comprised of more fundamental fermionic constituents. If the Higgs were to prove an elementary scalar, then it would be the first we have seen in nature. Conversely, if it were to prove composite, then a wealth of new physics beyond the Standard Model awaits. Either answer is compelling.

Of course, as with asking whether the Higgs is that of the Standard Model, the question cannot be absolutely answered with finite precision; we can only hope to learn that the Higgs is elementary down to experimentally accessible length scales. So perhaps the experimentally meaningful version of the question is: "Is the Higgs the most elementary scalar we have seen in nature?"

How do we quantify "most elementary"? One relative measure of compositeness is the ratio between a particle's physical size (as measured by its form factors) with its Compton wavelength. In some sense, this tells us how much of the particle's mass comes from compositeness itself. By this measure, the neutral pion is the most "elementary" of all the composite scalars yet seen in nature; $r_{\pi} / \lambda_{\pi} \sim m_{\pi} / m_{\rho} \sim 0.2$. In contrast, for the $\eta$, say, we have $r_{\eta} / \lambda_{\eta} \sim m_{\eta} / m_{\rho} \sim 0.7$. So what about the Higgs? We currently probe the "size" of the Higgs by looking for irrelevant operators that correct the Standard Model such as $\mathscr{O}_{H}=\frac{1}{2 \Lambda^{2}}\left(\partial_{\mu}|H|^{2}\right)^{2}$. This "size" operator looks like a shift in the wavefunction renormalization of the physical Higgs $h$ in the broken phase, which uniformly modifies all Higgs couplings to Standard Model particles.

While it is formally impossible to bound this operator in a model-independent fashion without directly measuring the width of the Higgs, it can be constrained with LHC data in a combined fit using only modest assumptions. At present, LHC bounds on $\mathscr{O}_{H}$ put $r_{H} / \lambda_{H} \lesssim 0.25$; we are currently probing pion-like levels of compositeness at the LHC. But here the LHC has the capacity to say much more; ultimately the HL-LHC will push far beyond this to set a bound $\Lambda \gtrsim 1.5 \mathrm{TeV}$ on the scale appearing in $\mathscr{O}_{H}$ [2]. This corresponds to $r_{H} / \lambda_{H} \lesssim 0.08$, allowing us to probe well beyond levels of pion-like compositeness and clearly determine whether the Higgs is the most elementary scalar we have encountered. Thus the LHC is capable of addressing this fundamental question, and the critical time for this measurement lies ahead of us.

\subsection{A self-interacting particle?}

At its face, this seems like a silly question; many particles in the Standard Model interact "with themselves". But the Standard Model Higgs raises a unique prospect: it is the only elementary particle predicted to interact with itself without changing any intrinsic quantum numbers. Even gluons change colors when they interact, thanks to the antisymmetry of the QCD structure constants. Thus the observation of Higgs self-interactions would be unlike anything yet seen in nature, a fascinating result even if ultimately in perfect agreement with Standard Model predictions. And, of course, the observation of Higgs self-interactions whose strength differs from the Standard Model prediction would point to a wealth of unforeseen new physics.

At present, bounds on di-Higgs production of any sort (including contributions not involving Higgs self-interactions) are much weaker than the Standard Model prediction, but this is expected 


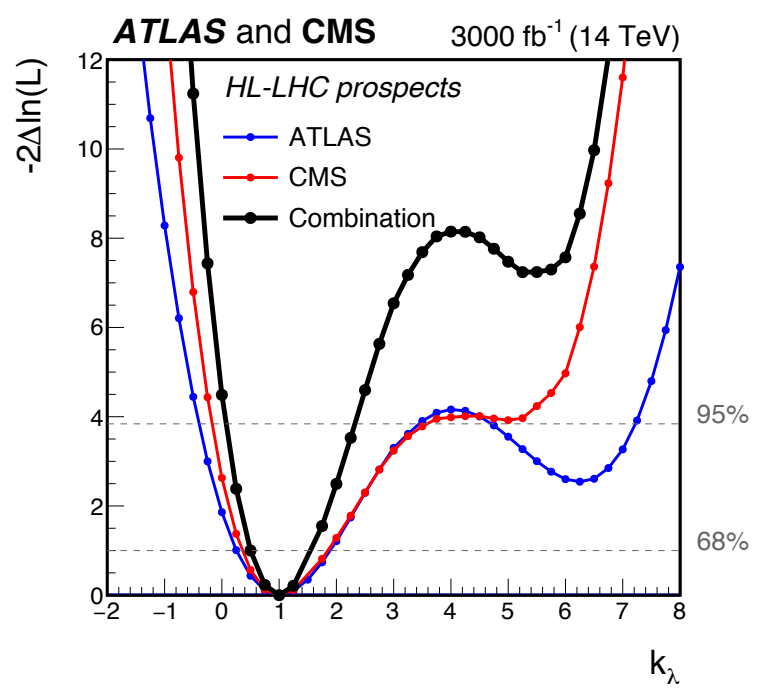

Figure 1: Minimum negative log-likelihood as a function of $\kappa_{\lambda} \equiv \lambda_{h h h} / \lambda_{h h h}^{\mathrm{SM}}$ from the projected combination of ATLAS and CMS searches for $h h$ production at the HL-LHC. The blue, red, and black lines correspond to the ATLAS, CMS, and combined likelihoods, respectively. Figure reproduced from [3].

to change substantially during the HL-LHC era. Ultimately, the combination of measurements by ATLAS and CMS in a variety of final states is expected to achieve $4 \sigma$ evidence for $h h$ production [3]. Assuming the the Standard Model signal, this will exclude the possibility that the Higgs has no self-coupling at the $\sim 2 \sigma$ level, a clear demonstration of the first fundamental self-interaction seen in nature. Once again, the LHC is capable of addressing a fundamental question, and the critical time for the measurement lies ahead of us.

Beyond answering the broader question, the precise value of the Higgs self-coupling has deep implications. At the moment, the discovery of the Higgs only gives us rudimentary information about the potential responsible for electroweak symmetry breaking, telling us about the second derivative of the potential at its mininum. Measurement of the Higgs self-coupling will contribute to a global understanding of the Higgs potential, indicating whether electroweak symmetry breaking is akin to that of Landau-Ginzburg theory (as predicted in the Standard Model) or something more exotic. Although it may sound hyperbolic, a sufficiently precise measurement of the Higgs self-coupling will also tell us the ultimate fate of the universe: the assuming only the presence of fields in the Standard Model, the Higgs self-coupling can run to negative values at higher energies, leading to metastability or absolute instability of the vacuum in which electroweak symmetry is broken. Precision measurement of the Higgs self-coupling, in conjunction with precision measurements of other Standard Model couplings, thus tells us whether our vacuum is stable, unstable, or metastable, and in the latter case even allows us to predict its lifetime.

\subsection{A Yukawa force mediator?}

No less interesting are the couplings of the Higgs to Standard Model fermions. Assuming it couples to fermions as the Standard Model predicts, the Higgs mediates a Yukawa force between fundamental particles. This is yet another phenomenon that we have never before encountered in 
nature, for the known Yukawa forces are all mediated by and between composite degrees of freedom. Considerable progress has recently been made towards establishing the Higgs as a Yukawa force-mediator, thanks to the $>5 \sigma$ observation of $t t H, H \rightarrow b b$ and $H \rightarrow \tau \tau$ processes during LHC Run $2[4,5,6,7,8,9]$. The significance of these measurements was perhaps best articulated by Gavin Salam in his theory vision talk at LHCP 2018 [10]: ${ }^{2}$

Is this any less important than the discovery of the Higgs boson itself? My opinion: no, because fundamental interactions are as important as fundamental particles.

- G. Salam, LHCP 2018

The measurement of Higgs couplings to tops, bottoms, and taus clearly establishes the Higgs as a Yukawa force-carrier between particles of the third generation. Yet the situation is no less interesting for the first and second generation - indeed, it arguably an even more significant target. It is the relative lightness of first- and second-generation fermions that makes the flavor puzzle compelling: the smallness of their Yukawa couplings in the Standard Model, albeit techically natural, cries out for a deeper explanation. It may be, for instance, that the Higgs only couples to light flavors through irrelevant operators, placing these couplings on a qualitatively different footing. Thus the measurement of Higgs couplings to light flavors remains a compelling target, one that could hold the key to the flavor puzzle.

Once again, while the current LHC dataset does not yet probe Higgs couplings to first- and second-generation fermions at the level of Standard Model predictions, the prospects at the HLLHC are promising. Both ATLAS and CMS collaborations should be capable of measuring the Higgs coupling to muons down to the $\sim 10-20 \%$ level assuming a Standard Model-like value [3], conclusively demonstrating that the Higgs mediates a Yukawa force between fermions of at least one second-generation species. The prospects for extending this evidence to second-generation quarks are somewhat more limited, but even here the combination of direct and indirect measurements should get within a factor of two of the Higgs coupling to charm. Thus the LHC is likewise capable of answering this fundamental question, with the first evidence recently garnered and perhaps the most interesting stage - the measurement of Higgs couplings to a second-generation fermion - still ahead of us.

Taken together, these three questions define a sharp and compelling physics program at the LHC that will largely come to fruition during the HL-LHC era. The outcome will be profound no matter what the answers hold, either uncovering predicted phenomena never before seen in nature, or pointing to a wealth of physics beyond our current picture.

\section{Looking beyond}

While the above questions about the properties of the Higgs are profoundly compelling and assured, pushing the frontiers of particle physics has never been solely about guaranteed outcomes, and there are a host of reasons to expect additional physics beyond the Standard Model. For the most part, these involve the same questions that came to the fore 35 years ago, and persist to this day.

${ }^{2}$ If I have learned anything in my career, it is to listen to Gavin. 


\subsection{The Naturalness Strategy}

The discovery of the Higgs boson cast considerable light on the mechanism of electroweak symmetry breaking: it is due, at least in large part, to the vacuum expectation value of an apparently fundamental scalar. But this leaves unanswered why electroweak symmetry breaking occurs - if the Higgs is an elementary scalar coming from an electroweak doublet, why is the mass-squared negative? And what sets the scale? These questions are often lumped together under the umbrella of the "electroweak hierarchy problem," but it is useful to think of them as very broad questions that are somewhat independent of our theoretical expectations. In any event, we are at an interesting time with respect to the hierarchy problem: dominant paradigms of the past forty years are now under increasing tension with null results from the LHC, which understandably has led to questions about the robustness of the hierarchy problem itself. The issue is exacerbated by the language we often use to discuss the hierarchy problem - quadratic divergences, UV sensitivity, Wilsonian expectations, and the lot - which can make the problem seem overly philosophical and perhaps questionably motivated.

Here I would like to articulate a different way of thinking about the hierarchy problem, one which is rooted in observation and a certain pragmatism about the value of theory to experiment. To understand this approach to thinking about the hierarchy problem, it is helpful to begin not with the Higgs boson, but with a much more familiar example: the electron.

We all secretly encountered the hierarchy problem early in our education as physicists when we learned about the self-energy of the electron in classical electrodynamics. ${ }^{3}$ This self-energy consists of two parts: a piece from the "bare" mass of the electron, and a piece from its electrostatic field. For an electron of "radius" $a$ (neglecting spin), we have

$$
W=\left(m_{e} c^{2}\right)_{o b s} \sim\left(m_{e} c^{2}\right)_{\text {bare }}+\frac{e^{2}}{a}
$$

Experimentally, we know $a \lesssim 10^{-18} \mathrm{~cm}$, so this apparently corresponds to a contribution to the self-energy of $\sim 100 \mathrm{GeV}$ and hence an absurd cancellation of the form

$$
0.511 \mathrm{MeV}=(-99999.489+100000.000) \mathrm{MeV}
$$

Now, one might hope that quantum mechanics automatically steps in to fix this apparent tuning (and indeed, quantum mechanics is often vaguely invoked in waving away the problem when we first encounter it), but in fact it makes the problem worse. In a quantum theory the fluctuations of the electromagnetic field in the "volume" $\sim a^{3}$ of a finite-sized electron are of the order $E^{2} \sim \frac{h c}{a^{4}}$ with a mean frequency of order $v \sim c / a$. This induces the electron to vibrate with an amplitude $x \sim e E / m v^{2}$ and an energy $\sim \frac{e^{2} E^{2}}{m v^{2}} \sim \frac{e^{2} h}{m c a^{2}}$. A more precise calculation gives $W_{\text {fluct }}=\frac{2 e^{2} \hbar}{m c a^{2}}$ which is larger than the classical self-energy by another seven orders of magnitude. (Note that this is but one of several additional contributions of a comparable size in the quantum theory.)

There is, however, another possibility that would avoid such a finely tuned cancellation: the theory could change at distances much larger than $a$ so that every contribution to the self-energy

\footnotetext{
${ }^{3}$ I first encountered the electron self-energy analogy for the hierarchy problem in Hitoshi Murayama's excellent ICTP summer school lecture notes [11], building on an earlier talk [12]. The somewhat elaborated version given here is based on the original calculation by Weisskopf [13].
} 


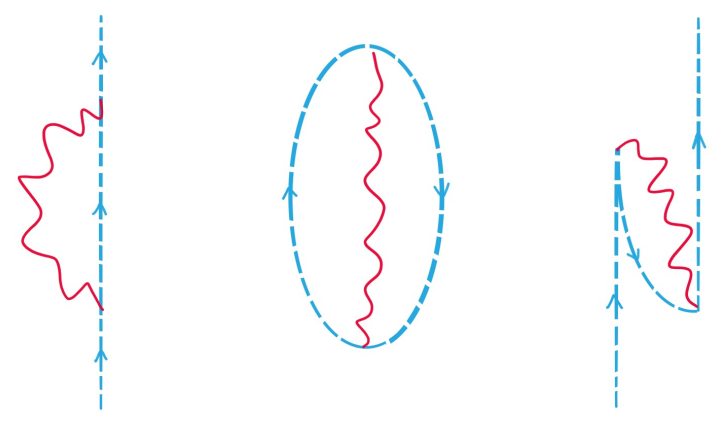

Figure 2: Left: Old-fashioned perturbation theory diagram for the one-loop correction to the electron selfenergy. Middle: Vacuum loops of electrons and positrons, the sort of phenomenon that changes the calculation on scales of order the Compton wavelength of the electron. Right: Old-fashioned perturbation theory diagram for the one-loop correction to the electron self-energy involving the positron.

is of the same order as the observed value, rendering the outcome "natural." To avoid fine tuning, we need the theory to change at scales on the order of $2 \times 10^{-13} \mathrm{~cm}$ in such a way as to alter the calculation of the self-energy. And indeed, this is what ultimately happens in nature. As Dirac taught us, the relativistic theory of the electron requires the addition of a new ingredient, the positron (more precisely, it is demanded by a new symmetry of the quantum theory, CPT). Now the theory has vacuum diagrams with both positrons and electrons, which modify the theory on length scales of order (twice the) the Compton wavelength of the electron,

$$
d \sim c \Delta t \sim \frac{c \hbar}{\Delta E} \sim \frac{\hbar}{2 m_{e} c} \sim 200 \times 10^{-13} \mathrm{~cm}
$$

The physical picture is that vacuum loops of electrons and positrons orient themselves around the bare electron charge in such a way that smooths out the charge distribution on scales of order the Compton wavelength. Just plugging this in gives the shift in self-energy of order $\Delta W \sim \frac{e^{2}}{h c} m_{e} c^{2}$. One can compute the one-loop self-energy of the electron in the theory with both electrons and positrons. The relevant loops correspond to linear divergences (in energy) from integrals of the form $\int d^{4} p / p^{3}$. The diagram containing the positron cancels the divergent contribution from the diagram with the electron, and the two leave behind a result that is only logarithmically dependent on $a[13]$ :

$$
\Delta W=+\frac{3}{2 \pi} \frac{e^{2}}{h c} m_{e} c^{2} \log \frac{\hbar}{m_{e} c a}+\ldots
$$

which is in agreement with our naive result up to some factors of $2 \pi$ related to virtual processes, and the logarithm.

Any time two large contributions cancel to extraordinary precision, there is usually a symmetry principle at work. In this case, it is chiral symmetry. The relativistic theory of the electron combines the electron and positron into a four-component Dirac fermion, which you can think of as combining two two-component Weyl fermions. When the mass of the Dirac fermion vanishes, the theory enjoys two separate symmetries corresponding to rotations of each of the Weyl fermions. Quantum corrections respect the symmetries of the quantum theory, so if the fermions are massless, quantum corrections will not change this. 
Now if the mass is nonzero, the action of the theory is only invariant under a linear combination of the two rotations; the other combination is broken by the mass. But quantum corrections to the mass must still know about the symmetry breaking, and so any corrections to the mass are proportional to the mass itself: $\delta m \propto m$. This implies they can only depend at most logarithmically on another scale, precisely what we see.

To reiterate the lesson, which I like to call the Naturalness Strategy: apparent fine-tuning between contributions to an observable are avoided by the picture changing such that these contributions are of the same order as the observable; this change of picture is typically associated with new degrees of freedom.

As you go up through the particle spectrum of the Standard Model, the Naturalness Strategy continues to be an effective one. For example, the electromagnetic coupling of the charged pion breaks the shift symmetry otherwise enjoyed by the pions, giving rise to a contribution to its selfenergy that diverges with its radius. Expressed in natural units, the correction to the charged pion mass is $\delta m_{ \pm}^{2}=\frac{3 \alpha}{4 \pi} \Lambda^{2}$ where $\Lambda \sim 1 / a$. The neutral pion does not possess analogous diagrams, so we may interpret the radiative correction in terms of a contribution to the charged-neutral pion mass splitting. The mass splitting is measured, so we can ask if our general logic about fine-tuning still applies. Avoiding tuning requires

$$
m_{ \pm}^{2}-m_{0}^{2}=(35.5 \mathrm{MeV})^{2} \rightarrow \Lambda \lesssim 850 \mathrm{MeV}
$$

And, indeed, the picture changes before you reach this point. One discovers that the pion has a finite size which cuts off the problematic diagrams at $1 / d \sim 770 \mathrm{MeV}$. This size is about a factor of 5-6 smaller than the Compton wavelength of the pion, and corresponds to the appearance of a whole zoo of particles, bound states of the strong interactions, which tell us that the pions are composite particles.

Thus the Naturalness Strategy appears to be an effective approach to anticipating new physics as we move up through the Standard Model particle spectrum, at least until we hit the Higgs. A scalar, the Higgs recapitulates the same issue encountered by the charged pion. Couplings of the Higgs to Standard Model fields appear to break any possible shift symmetries, and give rise to quantum corrections to the Higgs self-energy that are dominated by the largest couplings breaking the shift symmetry:

$$
\delta m_{H}^{2}=\frac{\Lambda^{2}}{16 \pi^{2}}\left[-6 y_{t}^{2}+\frac{9}{4} g_{2}^{2}+\ldots\right]
$$

Applying the Naturalness Strategy, we expect the picture to change around $\Lambda \lesssim 500 \mathrm{GeV}$ so that $\delta m_{H}^{2} \sim\left(m_{H}^{2}\right)_{o b s}$. Of course, this is just a restatement of the electroweak hierarchy problem, but I find it much more valuable to frame it as a strategy for looking for new physics that is grounded in examples encountered throughout the Standard Model itself. Moreover, by calling it a strategy, we are making clear what it is (and is not): a strategy for looking for physics beyond the Standard Model in an otherwise featureless landscape of possibilities. Strategies may succeed, or they may fail, but there are lessons in both outcomes.

Crucially, the Naturalness Strategy tells us to expect the picture to change at some scale, but it doesn't specify precisely how the picture changes; the specific mechanisms at work in enforcing 
the naturalness of the electron and the charged pion are different. Thus it bears emphasizing that realizations of the Naturalness Strategy are up to us.

To the extent that this leaves a vast landscape of possibilities, we've refined this strategy over the course of the past forty years using some perfectly sensible rules of thumb. These include:

1. The Standard Model coupled to gravity is a generic effective field theory (EFT).

2. The solutions to the hierarchy problem involve symmetries, low cutoffs, or anthropics.

3. Symmetries imply new particles charged under the Standard Model.

This has led to the current state of LHC searches associated with the hierarchy problem, focused primarily on partners of Standard Model particles charged under QCD that arise in symmetry-based solutions. At present, the main issue for these solutions is that the LHC has set limits on new states in the above frameworks on the order of $\mathrm{TeV}$ - few $\mathrm{TeV}$, leading to quantum corrections to the Higgs mass on the order of 100 times larger than observed parameter itself. It could be that the Naturalness Strategy has failed us, but it seems equally likely that the mechanism at work in this case is quite different from those that we have encountered before. The challenge is then to figure out what other phenomena might be hinted at by the Naturalness Strategy, and determine their experimental implications.

In the past five years, there has been considerable progress in pursuing solutions to the hierarchy problem that relax one or more of the above rules of thumb. While we do not have time to review all of these ideas, I would like to briefly focus on several developments that highlight the consequences of relaxing each of the above rules of thumb in turn. While none of these examples is likely the correct description of nature, they illustrate how much stands to be gained by venturing beyond established paradigms. If nothing else, we are in early days of exploring alternatives to conventional approaches, and there is no telling what further exploration might yield.

\subsubsection{Discrete symmetries}

The third, and most restrictive, rule of thumb is that symmetry solutions to the hierarchy problem imply new particles charged under the Standard Model, appearing at the scale $\Lambda$ implied by the Naturalness Strategy. It is this rule of thumb that has placed approaches to the hierarchy problem under the greatest tension with data, as partner particles charged under QCD can be abundantly produced at the LHC. Of course, as rules of thumb go, it's a very sensible one. In our most robust approaches to the hierarchy problem based on supersymmetry or global symmetry, the symmetry protecting the Higgs commutes with the Standard Model gauge group, implying that new particles associated with the symmetry carry the same gauge quantum numbers as their Standard Model counterparts.

This close association between symmetry-based solutions to the hierarchy problem and new colored particles can be broken, however, by discrete symmetries. While theories based on discrete symmetries do not have the same power as continuous ones - in particular, they still must be completed by supersymmetry or compositeness at higher scales - they allow for new physics appearing at $\Lambda$ to be partly or entirely neutral under the Standard Model. The archetypal example of this is the Twin Higgs [14], invented 14 years ago and generalized more recently into the broader program of 
neutral naturalness [15] based on discrete symmetries. The role of discrete symmetries here is to relate the Standard Model to a mirror copy via a $\mathbb{Z}_{2}$ exchange symmetry. The $\mathbb{Z}_{2}$ symmetry ensures that radiative corrections to the Standard Model Higgs doublet $H$ and its mirror copy $H^{\prime}$ enjoy an accidental $S U(4)$ symmetry arising from the $S U(2)$ symmetry of each doublet and the $\mathbb{Z}_{2}$ relating the couplings and spectrum. The one-loop self-energy of the Higgses now takes the form

$$
\Delta V=-\frac{6 y_{t}^{2}}{16 \pi^{2}} \Lambda^{2}\left(|H|^{2}+\left|H^{\prime}\right|^{2}\right)+\ldots
$$

Assuming the tree-level potential of $H, H^{\prime}$ enjoys an approximate $S U(4)$ symmetry (as is assured in a supersymmetric UV completion [16, 17]), then the observed Higgs boson is a goldstone of the approximate $S U(4)$, despite the fact that the spectrum of states respects only a $\mathbb{Z}_{2}$. Much like the electron in the presence of the positron, the Goldstone direction's self-energy retains only a logarithmic sensitivity to its radius, the scale being made up by the mass splitting between Standard Model states and their mirror counterparts:

$$
\Delta m_{\hat{H}}^{2}=-\frac{6 y_{t}^{2}}{16 \pi^{2}} \Lambda^{2}+\frac{6 y_{t}^{2}}{16 \pi^{2}} \Lambda^{2}-\frac{6 y_{t}^{2}}{16 \pi^{2}}\left(m_{T}^{2}-m_{t}^{2}\right) \log \frac{\Lambda^{2}}{m_{T}^{2}}
$$

There are still a plethora of new particles appearing near the weak scale in this theory, as predicted by the Naturalness Strategy, but they are not charged under the Standard Model and therefore avoid copious production at the LHC. Rather, their signatures are quite novel, including the decay of the Higgs boson into long-lived particles [18]. While new colored particles inevitably appear in association with the UV completion of these models, they can enter at scales higher by $\sim g_{*} / g_{S M}$ $[17,19]$ (where $g_{*}$ is a potentially large coupling associated with the UV completion) compared to theories based solely on continuous symmetries.

\subsubsection{Relaxion}

The second rule of thumb is largely a product of historical contingency: the hierarchy problem can be solved with reference to symmetries, low cutoffs, or anthropics. The first two are direct analogies with the electron and the pion, while the third has a storied history of application to astrophysical and cosmological problems [20]. If symmetry solutions are strongly constrained by LHC data, low cutoff solutions are even more strongly constrained, given the excellent agreement of physics at the weak scale with Standard Model predictions. Anthropic explanations, while intriguing, are often challenging to convert into sharp experimental predictions. But the introduction of the relaxion $[21]^{4}$ opened a fourth avenue for approaching the problem. The basic idea here is for the evolution of a light field $\phi$ in the early universe to scan the Higgs mass (via, say, a coupling $g \phi|H|^{2}$ ), coming to rest where the sum of contributions to the Higgs mass (from the vacuum expectation value of the scanning field, and the various contributions to the Higgs self-energy) were close to zero. In the case of the relaxion, $m_{H}^{2}=0$ is made special by the contribution of electroweak symmetry breaking to the masses of quarks. If the relaxion is an axion-like particle (in the sense of coupling to the $G \tilde{G}$ combination of QCD field strengths), these quark masses contribute to the amplitude of a cosine potential that grows with the Higgs vacuum expectation value. Thus if the

\footnotetext{
${ }^{4}$ For a related predecessor, see [22].
} 
relaxion is rolling (along, say, a gentle linear potential) in such a way that the Higgs mass-squared is scanned from positive to negative values, growing bumps due to the cosine potential will appear as the $\phi$ field crosses through the point where $m_{H}^{2}=0$. Assuming the energy stored in the relaxion can be dissipated efficiently (via inflation in the original proposal), it will stop near the onset of these bumps and explain the smallness of the weak scale.

The main challenge of the relaxion is that it may transmogrify well-understood naturalness problems into less concrete issues involving trans-Planckian field excursions, low-scale inflation, and the like. At the very least, these are qualitatively different problems from conventional approaches to the hierarchy problem, and may have correspondingly original solutions. From an experimental perspective, the most interesting aspect of the relaxion is that it generically predicts experimental signatures associated with Higgs-relaxion mixing. While the scanning coupling $g \phi|H|^{2}$ can be small enough to evade experimental detection, the dependence of the bumps in the relaxion potential on the Higgs - schematically, $V(\phi, H) \supset \Lambda_{\text {bumps }}^{4}(H) \cos (\phi / f)$ - must necessarily be large to effectively stop the relaxion, leading to significant mixing between $\phi$ and $H$. This in turn gives a variety of experimental signatures that vary in detail with the relaxion mass [23], which depart considerably from signatures associated with conventional solutions.

\subsubsection{UV/IR mixing}

The first rule of thumb is, in some sense, the most inevitable: the Standard Model itself is an effective field theory, and the principles of EFT have been remarkably successful in treating Standard Model phenomena. Indeed, the hierarchy problem itself is a problem of EFT, concerned as it is with generic expectations for the size of contributions to the Higgs self-energy. But this also points to another, far more radical, possibility: perhaps the resolution to the hierarchy problem is the breakdown of EFT itself. This is not quite as radical as it may sound; we already expect EFT to break down around the Planck scale. There are hints, moreover, that consistency of the theory of quantum gravity might also proscribe the space of effective field theories, in the sense of limiting the parameter space of EFTs that can be consistently coupled to gravity. This "Swampland Program" [24] has led to a profusion of activity developing the conjectured form of these limitations and some of their possible phenomenological consequences, including implications for the hierarchy problem [25].

Perhaps the sharpest possibility is raised by the Weak Gravity Conjecture (WGC) [26], which posits that an abelian gauge theory must contain a state of charge $q$ and mass $m$ satisfying

$$
q \geq \frac{m}{M_{\mathrm{Pl}}}
$$

Relative to many other conjectures in the Swampland Program, the WGC has the advantage that it can be motivated by largely infrared considerations (such as the decay of extremal black holes) rather than the details of known string constructions. Since the bound can be re-written as a bound on the mass of the state satisfying the WGC, it is tempting to use this to bound the value of the Higgs vacuum expectation value [27]. Although many assumptions are required to convert the WGC bound into a bound on the weak scale, successful realizations invariably require new particles at or below the weak scale with an appreciable coupling to the Higgs [28]. Thus even attempts to circumvent the Naturalness Strategy from violations of pure EFT considerations still give rise 

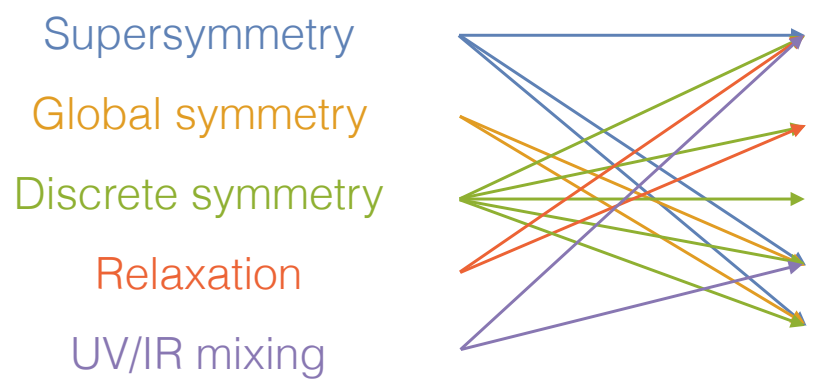
Higgs $\rightarrow$ invisible
Higgs $\rightarrow$ exotic
Higgs $\rightarrow$ LLPS
Higgs couplings
Di-Higgs

Figure 3: Higgs signatures of approaches to the hierarchy problem, conventional and otherwise.

to sharp predictions for new physics at the weak scale, at least in the context of the Weak Gravity Conjecture.

It may seem that the lesson of the above examples is "anything goes" [29] - that is, that there are no overriding signatures associated with the Naturalness Strategy once our historical rules of thumb are relaxed. Indeed, as we have seen, many of the classical signatures of naturalness - light, QCD-charged partners of the top quark, for example - are absent in the above examples. However, we are far from being unmoored in a sea of signatures. At the end of the day, any mechanism for addressing the hierarchy problem must couple to the Higgs, and it will leave its fingerprints on Higgs properties. Discrete symmetries leave their mark on invisible Higgs decays, exotic Higgs decays, Higgs couplings, and di-Higgs production. Relaxation leaves its mark on both invisible and exotic Higgs decays. UV/IR mixing leaves its mark on invisible Higgs decays and Higgs couplings. If anything, relaxing our historical rules of thumb increases the importance of the Higgs in pursuing the naturalness strategy, because modifications to Higgs properties appear to be an irreducible signature.

\subsection{Dark matter, strong $\mathrm{CP}$, and flavor}

Much of the discussion thus far about physics beyond the Standard Model has emphasized the hierarchy problem, insofar as the Naturalness Strategy predicts new signatures that are a prime target for the LHC. But the LHC may also play a key role in answering other pressing questions pointing to physics beyond the Standard Model - for example, the nature of dark matter, the apparent CP-invariance of the strong interactions, and the underlying structure of flavor.

Dark matter: The existence of dark matter has been established by a host of astrophysical and cosmological evidence, although this evidence alone only points to gravitational couplings between dark matter and the Standard Model. However, the near coincidence of the baryonic and dark matter abundances $\Omega_{b}$ and $\Omega_{d m}$ is highly suggestive of interactions beyond the gravitational, making dark matter a sharp target for terrestrial tests. Although the majority of these tests are being pursued through various forms of direct detection experiments, the LHC is strongly complementary to these efforts. Perhaps the LHC's greatest strength is its insensitivity to the dark matter mass, subject to assumptions; as long as the dark matter appears in an appreciable fraction of the decays of states 


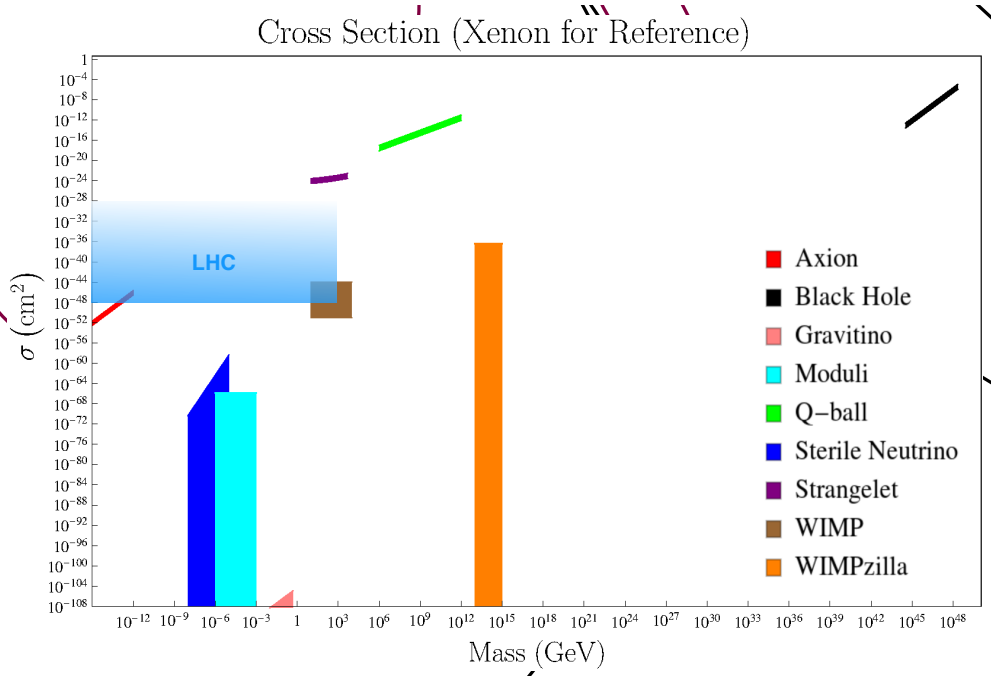

Figure 4: LHC sensitivity schematically overlaid on the range of dark matter candidates' masses and interaction cross sections. Although this sensitivity is ultimately model-dependent, it illustrates the LHC's significant reach in dark matter mass. Adapted from [30].

produced at the LHC (whether new mediators or Standard Model particles), the LHC can probe all dark matter masses below its kinematic threshold, with continuous improvement in sensitivity throughout the HL-LHC era. While the relation between these constraints and those coming from direct detection experiments is model-dependent, it highlights the sense in which the LHC provides a complementary approach to direct detection experiments with broad coverage in the space of masses and couplings.

It also bears emphasizing the role that the Higgs may play in the dark matter puzzle. If dark matter couples to the Standard Model through a weakly-interacting, weak-scale mediator, the Higgs is perhaps the best Standard Model candidate in light of constraints from direct detection experiments. Should this be the case, invisible decays of the Higgs into dark matter provide a powerful probe, competitive with direct detection experiments for dark matter masses in the tens of $\mathrm{GeV}$ and considerably stronger at lighter masses.

Strong CP: Why do the strong interactions appear to conserve CP, when it is notably violated by the weak interactions? To highlight the problem, it is helpful to first consider a sort of classical toy model consisting of the three valence quarks in the neutron. The relative arrangement of these quarks in the volume of the neutron gives rise to an electric dipole moment. Experimentally, the bound on the neutron electric dipole moment is $\left|d_{n}\right| \lesssim 3 \times 10^{-26} e \mathrm{~cm}$, which in this toy model implies that the up and down quarks must lie on a line to within an angle $\theta \lesssim 10^{-12}$. This seems like an astronomical tuning! Of course, the quantum version of the problem is far more subtle; at its heart, the naively $\mathscr{O}(1) \mathrm{CP}$-violating $\theta$ parameter of the strong interactions is constrained by the neutron EDM bound to be $\theta<10^{-10}$.

We don't typically discuss the strong CP problem at the LHC because the most popular solution, the QCD axion, is better constrained by non-collider tests. However, there are other solutions whose strengths and weaknesses are comparable to the axion solution and provide a prime target 
for the LHC. One such class of eamples are parity solutions [31] extending the Standard Model to include left-right parity, $S U(3)_{c} \times S U(2)_{L} \times U(1)_{Y} \rightarrow S U(3)_{c} \times S U(2)_{L} \times S U(2)_{R} \times U(1)_{Y}$. These theories predict new parity partners of all SM fermions that are likely LHC accessible and can be probed by vector-like quark searches [32]. Thus the LHC can play a role in shaping our understanding of the strong $\mathrm{CP}$ problem, a possibility that perhaps warrants more attention.

Flavor: In many respects, the success of the Standard Model flavor structure has long been a source of discomfort for beyond-the-Standard Model physics, in the sense that generic extensions of the Standard Model violate this flavor structure and must either be decoupled in energy or complicated significantly in order to regain agreement with precision flavor tests. Now, however, we find ourselves in an era of numerous flavor anomalies, particularly involving fermions of the 3rd generation. While it is too early to tell whether these hints will ultimately point to the breakdown of the Standard Model, decisive input from Belle II and the combination of ATLAS, CMS, and LHCb during the HL-LHC era will point the way. For example, LHCb measurements by 2025 and the subsequent Upgrade II have the potential to significantly improve the significance of current tensions in $b \rightarrow c \tau v$ [33]; the combination of ATLAS, CMS, and LHCb have comparable potential to improve the significance of tensions in $b \rightarrow s \mu^{+} \mu^{-}$[33]; and LHCb Upgrade II will significantly sharpen the measurement of weak $\mathrm{CP}$ violation in the charm sector.

\subsection{Who ordered that?}

Thus far, I have focused on predictions for new phenomena motivated primarily by theoretical considerations. But the fact remains that most of the Standard Model was discovered not by following sharp theoretical predictions for new degrees of freedom, but simply by experimental exploration. Given this history, it seems likely that the discovery of physics beyond the Standard Model will result not from a line of theoretical argumentation, but instead from covering new ground. In this regard, it is worth emphasizing the value of new searches, new signatures, and new experimental opportunities capable of uncovering exotic phenomena that could be right under our noses, undiscovered only because we have not yet looked.

New (theorist-free) searches: Many of us live in fear of missing new physics at the LHC simply because we did not ask the right questions or define the right signal region. One way of alleviating this fear is to develop new searches that are as independent of theory prejudices as possible. For example, by focusing on simplified topologies wholly independent of theory motivation, it is possible to identify bumps in various invariant mass distributions that are not subject to any published LHC constraints but are amenable to straightforward analyses [34, 35]. Another option is to replace theorists with machines, e.g. through weakly supervised or unsupervised learning for classification and anomaly detection [36, 37, 38, 39, 40, 41, 42, 43, 44, 45, 46, 47]. Although we have some ways to go before these techniques supplant existing search strategies, it is clear that they offer new methods for ensuring that no stone goes unturned at the LHC.

New signatures: Perhaps the most compelling possibility is for physics beyond the Standard Model to manifest itself via qualitatively new signatures that, once the detector response is appropriately quantified, are readily amenable to discovery. Among the greatest sources for these 
signatures are long-lived particles (LLPs), which can give rise to exotic signatures such as disappearing or kinked tracks; displaced multi-track vertices; displaced leptons, lepton-jets, or dilepton pairs; trackless, low-EMF jets; anomalous energy deposition; emerging jets; and non-pointing photons. The identification and coverage of these scenarios has increased significantly in the course of the last decade, and one of the most impressive lessons has been that limits on a given scenario can rapidly progress from nonexistent to comprehensive once the opportunity is identified. Nonetheless, searches for these exotic signatures remains largely resource-limited at the LHC, and there remains a great deal of low-lying fruit, with excellent opportunities for close interplay between theory and experiment [48].

New opportunities: The planned and potential hardware upgrades associated with the LS2 and LS3 shutdowns will open up sensitivity to blind spots in current LHC coverage. This is perhaps best exemplified by searches for exotic Higgs decays into long-lived particles with hadronic final states when the average LLP decay length lies between $1 \mathrm{~cm}-1 \mathrm{~m}$. At present, this is essentially unconstrained by existing LLP searches, in large part due to the lack of efficient trigger strategies over these decay lengths. However, there are not one but three potential upgrades that could improve limits by orders of magnitude: proposed displaced track triggers [49, 50], precision timing triggers [51], and a suite of planned LHCb upgrades [52]. To the extent that the final state can be made essentially background-free with high signal efficiency, these potential or planned improvements will improve sensitivity to highly motivated signals of new physics by as much as five orders of magnitude - an almost unheard-of level of improvement at this stage of the LHC.

New force multipliers: Recent years have seen a profusion of proposals for auxiliary detectors that can work in tandem with existing LHC detectors and collision points to provide sensitivity to otherwise hopeless scenarios. In this respect, they serve as force multipliers, combining with existing detectors to provide sensitivity unavailable to either in isolation. Typically these auxiliary detectors are physically shielded from the relevant collision points, significantly reducing Standard Model backgrounds to exotic signatures. Proposals at varying degrees of maturity include MilliQan [53], FASER [54], CODEX- $b$ [55], and MATHUSLA [56] which provide sensitivity to a range of millicharged or long-lived new particles poorly constrained by existing detectors. The scope of possible auxiliary experiments is vast [57], and further development in the next decade may lend the LHC sensitivity to a host of new signatures.

\section{Future colliders}

As we have seen, the LHC promises to make considerable progress in answering fundamental questions surrounding the Higgs, as well as a host of longer-standing questions involving the underpinnings of the Standard Model. But the ultimate answers to questions surrounding the Higgs inevitably lie in proposed colliders such as CEPC [58, 59], FCC-ee [60], the ILC [61, 62], and CLIC $[63,64]$ that would operate in part as Higgs factories. By achieving sensitivity to deviations from Standard Model Higgs couplings at the sub-percent level, these colliders would allow us to probe virtual corrections from beyond-the-Standard Model physics to tree-level processes in the Standard Model, bringing qualitatively new power to the search for new physics. 
While the details of each proposal vary, each ultimately offers an order of magnitude improvement (over the already-impressive HL-LHC sensitivity) in measurements of Higgs properties most interesting for the Standard Model and beyond. For example, the combination of the HL-LHC dataset with data from the ILC, CLIC, CEPC, or FCC-ee would improve sensitivity to the Higgs size operator $\mathscr{O}_{H}$ to the point of probing $r_{H} / \lambda_{H} \lesssim 0.03-0.04$, testing Higgs compositeness to nearly an order of magnitude beyond that of the pion [2]. They would also provide sensitivity to untagged decays of the Higgs down to branching ratios at the percent level and invisible decays of the Higgs well below the percent level, with sweeping implications for physics beyond the Standard Model. In this respect, these proposed colliders are the ideal machines for the questions of our era, and now is the time to seriously contemplate their construction.

Of course, any discussion of future colliders beyond the LHC must grapple with the reality that no guaranteed discoveries lie ahead of us. But this begs the question: does the physics case for future colliders require a guarantee of discovery? It is easy to look back on the physics case for the LHC and assume that the guarantee of Higgs discovery was essential to its approval, but this is a considerable oversimplification. Looking back to the 1984 ECFA-CERN Workshop, C.H. Llewellyn Smith's comments on the prospects for Higgs discovery at the LHC are quite illuminating [1]:

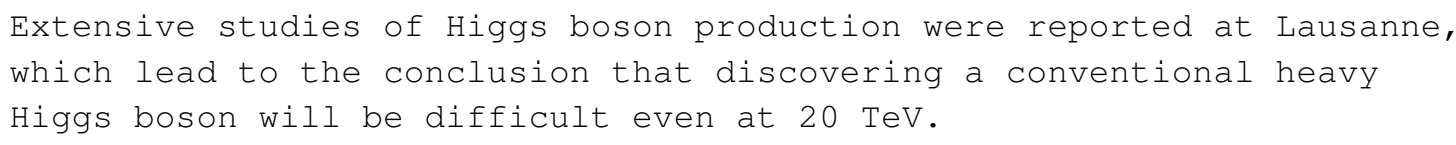

The same point reappears in the organizers' summary report of the same proceedings,

On the other hand, searching for the Higgs meson as it appears in the standard model looks difficult.

Far from being central to the initial physics case of the LHC, the discovery of the Higgs was viewed as a remote possibility despite its kinematic accessibility. And yet the case for the LHC was strong even then. If nothing else, this is a reminder that we build colliders not to confirm what we already know, but to explore what we do not.

\section{Conclusions}

We began with the theory vision from the inception of the LHC, and so it is perhaps fitting to end with inspiration from the same 1984 proceedings. In his concluding remarks, C.H. Llewellyn Smith surveyed proposals for new phenomena accessible at the then-future LHC and arrived at the following assessment:

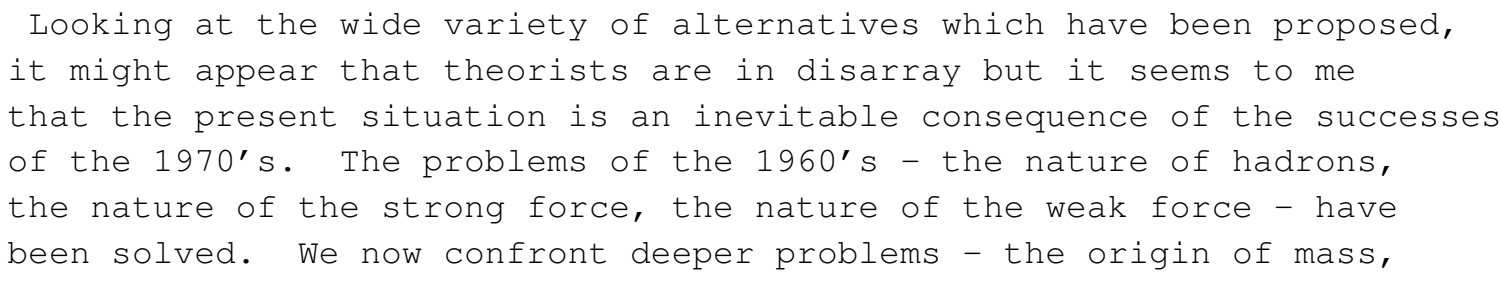




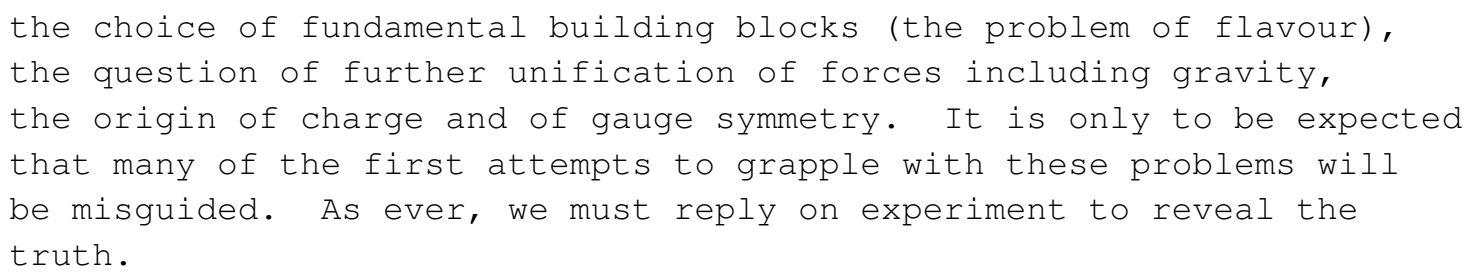

In the 35 years since these remarks, the LHC has done a great deal to confront these deeper problems. It has cast light on the origin of mass, raising a whole host of new questions surrounding the nature of the Higgs. Null results in searches for new phenomena have proved equally enlightening by suggesting which first attempts to grapple with the problems of the Standard Model might be misguided. Yet the failure of first attempts is nothing more than an invitation to renewed effort. The questions themselves remain robust, and their urgency only grows with time.

As I have tried to argue here, the Higgs discovery defines new conceptual questions: Is it the first fundamental scalar? The first self-interacting particle? The first Yukawa force-carrier between fundamental particles? These are the questions of this era. Thankfully, we are poised to make substantial progress on them throughout the lifetime of the LHC and decisively answer them with a future collider program. The LHC also provides compelling opportunities to address the longerstanding questions of the weak scale, including the reason for electroweak symmetry breaking (and the scale thereof); the underpinnings of flavor; the nature of dark matter; and the mystery of strong $\mathrm{CP}$ conservation. With a host of new ideas, new tools, and new opportunities, the future of the LHC is incredibly bright. Data disfavoring first attempts to address the problems of the Standard Model should not be a source of discouragement, but a source of encouragement: it is pointing us in new directions and telling us which prejudices to shed. It is a time of exploration for both theory and experiment. Now, as ever, we must rely on experiment to reveal the truth.

\section{Acknowledgments}

This work is supported in part by the US Department of Energy under the Early Career Award DE-SC0014129 and the Cottrell Scholar Program through the Research Corporation for Science Advancement.

\section{References}

[1] M. Jacob, ed., Proceedings, ECFA-CERN Workshop on large hadron collider in the LEP tunnel, 1984.

[2] J. de Blas et al., Higgs Boson Studies at Future Particle Colliders, arXiv:1905.03764.

[3] HL/HE WG2 group Collaboration, M. Cepeda et al., Higgs Physics at the HL-LHC and HE-LHC, arXiv:1902.00134.

[4] CMS Collaboration, A. M. Sirunyan et al., Observation of the Higgs boson decay to a pair of $\tau$ leptons with the CMS detector, Phys. Lett. B779 (2018) 283-316, [arXiv:1708.00373].

[5] ATLAS Collaboration, M. Aaboud et al., Cross-section measurements of the Higgs boson decaying into a pair of $\tau$-leptons in proton-proton collisions at $\sqrt{s}=13 \mathrm{TeV}$ with the ATLAS detector, Phys. Rev. D99 (2019) 072001, [arXiv:1811.08856]. 
[6] CMS Collaboration, A. M. Sirunyan et al., Observation of $\mathrm{t} \overline{\mathrm{t}} H$ production, Phys. Rev. Lett. 120 (2018), no. 23 231801, [arXiv:1804.02610].

[7] ATLAS Collaboration, M. Aaboud et al., Observation of Higgs boson production in association with a top quark pair at the LHC with the ATLAS detector, Phys. Lett. B784 (2018) 173-191, [arXiv:1806.00425].

[8] ATLAS Collaboration, M. Aaboud et al., Observation of $H \rightarrow b \bar{b}$ decays and $V H$ production with the ATLAS detector, Phys. Lett. B786 (2018) 59-86, [arXiv : 1808 . 08238].

[9] CMS Collaboration, A. M. Sirunyan et al., Observation of Higgs boson decay to bottom quarks, Phys. Rev. Lett. 121 (2018), no. 12 121801, [arXiv: 1808 .08242].

[10] G. P. Salam, Theory vision, PoS LHCP2018 (2018) 304, [arXiv : 1811 . 11282].

[11] H. Murayama, Supersymmetry phenomenology, in Proceedings, Summer School in Particle Physics: Trieste, Italy, June 21-July 9, 1999, pp. 296-335, 2000. hep-ph / 0002232.

[12] H. Murayama, Supersymmetry, in 22nd INS International Symposium on Physics with High-energy Colliders Tokyo, Japan, March 8-10, 1994, pp. 0357-380, 1994. hep-ph/ 9410285.

[13] V. F. Weisskopf, On the Self-Energy and the Electromagnetic Field of the Electron, Phys. Rev. 56 (1939) 72-85.

[14] Z. Chacko, H.-S. Goh, and R. Harnik, The Twin Higgs: Natural electroweak breaking from mirror symmetry, Phys. Rev. Lett. 96 (2006) 231802, [hep-ph/ 0506256 ].

[15] N. Craig, S. Knapen, and P. Longhi, Neutral Naturalness from Orbifold Higgs Models, Phys. Rev. Lett. 114 (2015), no. 6 061803, [arXiv: 1410.6808 ].

[16] S. Chang, L. J. Hall, and N. Weiner, A Supersymmetric twin Higgs, Phys. Rev. D75 (2007) 035009, [hep-ph/0604076].

[17] N. Craig and K. Howe, Doubling down on naturalness with a supersymmetric twin Higgs, JHEP 03 (2014) 140, [arXiv:1312.1341].

[18] N. Craig, A. Katz, M. Strassler, and R. Sundrum, Naturalness in the Dark at the LHC, JHEP 07 (2015) 105, [arXiv:1501.05310].

[19] R. Contino, D. Greco, R. Mahbubani, R. Rattazzi, and R. Torre, Precision Tests and Fine Tuning in Twin Higgs Models, Phys. Rev. D96 (2017), no. 9 095036, [arXiv: 1702.00797 ].

[20] B. Carter, Large number coincidences and the anthropic principle in cosmology, IAU Symp. 63 (1974) 291.

[21] P. W. Graham, D. E. Kaplan, and S. Rajendran, Cosmological Relaxation of the Electroweak Scale, Phys. Rev. Lett. 115 (2015), no. 22 221801, [arXiv: 1504.07551 ].

[22] G. Dvali and A. Vilenkin, Cosmic attractors and gauge hierarchy, Phys. Rev. D70 (2004) 063501, [hep-th/0304043].

[23] T. Flacke, C. Frugiuele, E. Fuchs, R. S. Gupta, and G. Perez, Phenomenology of relaxion-Higgs mixing, JHEP 06 (2017) 050, [arXiv: 1610.02025$].$

[24] C. Vafa, The String landscape and the swampland, hep-th/0509212.

[25] L. E. Ibanez, V. Martin-Lozano, and I. Valenzuela, Constraining the EW Hierarchy from the Weak Gravity Conjecture, arXiv:1707.05811. 
[26] N. Arkani-Hamed, L. Motl, A. Nicolis, and C. Vafa, The String landscape, black holes and gravity as the weakest force, JHEP 06 (2007) 060, [hep-th/ 0601001 ].

[27] C. Cheung and G. N. Remmen, Naturalness and the Weak Gravity Conjecture, Phys. Rev. Lett. 113 (2014) 051601, [arXiv:1402.2287].

[28] N. Craig, I. Garcia Garcia, and S. Koren, The Weak Scale from Weak Gravity, arXiv: 1904.08426.

[29] P. Feyerabend, Against Method. Verso, 1993.

[30] A. Kusenko and L. J. Rosenberg, Working Group Report: Non-WIMP Dark Matter, in Proceedings, 2013 Community Summer Study on the Future of U.S. Particle Physics: Snowmass on the Mississippi (CSS2013): Minneapolis, MN, USA, July 29-August 6, 2013, 2013. arXiv:1310.8642.

[31] K. S. Babu and R. N. Mohapatra, A Solution to the Strong CP Problem Without an Axion, Phys. Rev. D41 (1990) 1286.

[32] R. T. D'Agnolo and A. Hook, Finding the Strong CP problem at the LHC, Phys. Lett. $\mathbf{B 7 6 2}$ (2016) 421-425, [arXiv:1507.00336].

[33] A. Cerri et al., Opportunities in Flavour Physics at the HL-LHC and HE-LHC, arXiv:1812.07638.

[34] N. Craig, P. Draper, K. Kong, Y. Ng, and D. Whiteson, The unexplored landscape of two-body resonances, Acta Phys. Polon. B50 (2019) 837, [arXiv: 1610.09392$].$

[35] J. H. Kim, K. Kong, B. Nachman, and D. Whiteson, The motivation and status of two-body resonance decays after the LHC Run 2 and beyond, arXiv:1907.06659.

[36] L. M. Dery, B. Nachman, F. Rubbo, and A. Schwartzman, Weakly Supervised Classification in High Energy Physics, JHEP 05 (2017) 145, [arXiv: 1702.00414$].$

[37] T. Cohen, M. Freytsis, and B. Ostdiek, (Machine) Learning to Do More with Less, JHEP 02 (2018) 034, [arXiv:1706.09451].

[38] E. M. Metodiev, B. Nachman, and J. Thaler, Classification without labels: Learning from mixed samples in high energy physics, JHEP 10 (2017) 174, [arXiv: 1708.02949 ].

[39] E. M. Metodiev and J. Thaler, Jet Topics: Disentangling Quarks and Gluons at Colliders, Phys. Rev. Lett. 120 (2018), no. 24 241602, [arXiv: 1802.00008 ].

[40] A. Andreassen, I. Feige, C. Frye, and M. D. Schwartz, JUNIPR: a Framework for Unsupervised Machine Learning in Particle Physics, Eur. Phys. J. C79 (2019), no. 2 102, [arXiv: 1804.09720 ].

[41] J. H. Collins, K. Howe, and B. Nachman, Anomaly Detection for Resonant New Physics with Machine Learning, Phys. Rev. Lett. 121 (2018), no. 24 241803, [arXiv: 1805.02664 ].

[42] R. T. D’Agnolo and A. Wulzer, Learning New Physics from a Machine, Phys. Rev. D99 (2019), no. 1 015014, [arXiv:1806.02350].

[43] J. W. Monk, Deep Learning as a Parton Shower, JHEP 12 (2018) 021, [arXiv: 1807.03685 ].

[44] A. De Simone and T. Jacques, Guiding New Physics Searches with Unsupervised Learning, Eur. Phys. J. C79 (2019), no. 4 289, [arXiv: 1807.06038 ].

[45] J. Hajer, Y.-Y. Li, T. Liu, and H. Wang, Novelty Detection Meets Collider Physics, arXiv:1807.10261. 
[46] A. A. Pol, G. Cerminara, C. Germain, M. Pierini, and A. Seth, Detector monitoring with artificial neural networks at the CMS experiment at the CERN Large Hadron Collider, Comput. Softw. Big Sci. 3 (2019), no. 13 , [arXiv:1808.00911].

[47] M. Farina, Y. Nakai, and D. Shih, Searching for New Physics with Deep Autoencoders, arXiv:1808.08992.

[48] J. Alimena et al., Searching for Long-Lived Particles beyond the Standard Model at the Large Hadron Collider, arXiv:1903.04497.

[49] Y. Gershtein, CMS Hardware Track Trigger: New Opportunities for Long-Lived Particle Searches at the HL-LHC, Phys. Rev. D96 (2017), no. 3 035027, [arXiv: 1705.04321 ].

[50] CMS Collaboration, C. Collaboration, First Level Track Jet Trigger for Displaced Jets at High Luminosity LHC, .

[51] J. Liu, Z. Liu, and L.-T. Wang, Enhancing Long-Lived Particles Searches at the LHC with Precision Timing Information, Phys. Rev. Lett. 122 (2019), no. 13 131801, [arXiv: 1805.05957 ].

[52] Working Group 3 Collaboration, X. Cid Vidal et al., Beyond the Standard Model Physics at the HL-LHC and HE-LHC, arXiv: 1812.07831.

[53] A. Ball et al., A Letter of Intent to Install a milli-charged Particle Detector at LHC P5, arXiv:1607.04669.

[54] FASER Collaboration, A. Ariga et al., Letter of Intent for FASER: ForwArd Search ExpeRiment at the LHC, arXiv:1811.10243.

[55] V. V. Gligorov, S. Knapen, M. Papucci, and D. J. Robinson, Searching for Long-lived Particles: A Compact Detector for Exotics at LHCb, Phys. Rev. D97 (2018), no. 1015023 , [arXiv:1708.09395].

[56] MATHUSLA Collaboration, C. Alpigiani et al., A Letter of Intent for MATHUSLA: A Dedicated Displaced Vertex Detector above ATLAS or CMS., arXiv:1811.00927.

[57] J. Beacham et al., Physics Beyond Colliders at CERN: Beyond the Standard Model Working Group Report, arXiv:1901.09966.

[58] CEPC Study Group Collaboration, CEPC Conceptual Design Report: Volume 1 - Accelerator, arXiv:1809.00285.

[59] CEPC Study Group Collaboration, CEPC Conceptual Design Report: Volume 2 - Physics \& Detector, arXiv:1811.10545.

[60] TLEP Design Study Working Group Collaboration, M. Bicer et al., First Look at the Physics Case of TLEP, JHEP 01 (2014) 164, [arXiv:1308.6176].

[61] T. Behnke, J. E. Brau, B. Foster, J. Fuster, M. Harrison, J. M. Paterson, M. Peskin, M. Stanitzki, N. Walker, and H. Yamamoto, The International Linear Collider Technical Design Report - Volume 1: Executive Summary, arXiv:1306.6327.

[62] H. Baer, T. Barklow, K. Fujii, Y. Gao, A. Hoang, S. Kanemura, J. List, H. E. Logan, A. Nomerotski, M. Perelstein, et al., The International Linear Collider Technical Design Report - Volume 2: Physics, arXiv:1306.6352.

[63] M. Aicheler, P. Burrows, M. Draper, T. Garvey, P. Lebrun, K. Peach, N. Phinney, H. Schmickler, D. Schulte, and N. Toge, A Multi-TeV Linear Collider Based on CLIC Technology, .

[64] J. de Blas et al., The CLIC Potential for New Physics, arXiv: 1812.02093. 